\title{
A little more on travel times
}

\author{
Riccardo Rigon ${ }^{1}$ \\ ${ }^{1}$ University of Trento
}

January 11, 2022

Let's assume we have a domain $\Omega$ of boundaries $\partial \Omega$ as in Figure 1.

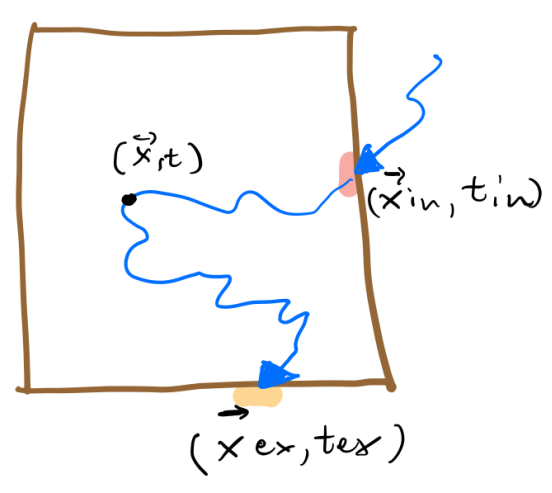

Figure 1: A domain and a water parcel that enters into it and eventually exit

A water parcel enters in it at time $t_{i n}$ at position $\vec{x}_{i n}$ and exits at time $t_{e x}$ and position $\vec{x}_{e x}$. Its travel time is, by definition $T:=t_{e x}-t_{i n}$. Let us define $s\left(t, \vec{x} ; t_{i n}, \vec{x}_{i n}\right)$ the volume (mass or moles, at convenience) of water parcels at position $\vec{x}$ at time $t$. A little daemon, She, collects all the parcels inside a neighborhood of $x \in \Omega$ (not in its boundary) at time time $t$ obtaining

$$
S(t, \vec{x}):=\int_{\partial \Omega^{+}} \int_{-\infty}^{t} s\left(t, \vec{x}, t_{i n}^{\prime},{\overrightarrow{x^{\prime}}}_{i n}\right) d t_{i n}^{\prime} d \vec{x}_{i n}
$$

where an integration over all the entering parcels and the injection time has been performed. The domain $\partial \Omega^{+}$ represents the boundaries location were the parcels entered the volume. $S(t, \vec{x})$ is the volume (mass, moles number) of water around $\vec{x}$. These parcels have entered $\Omega$ at different times (i.e. were injected in the control volume at different times) and therefore, have different ages. The probability of each age can be given by:

$$
p\left(t_{i n}, \vec{x}_{i n} \mid t, \vec{x}\right):=\frac{s\left(t, \vec{x}, t_{i n}, \vec{x}_{i n}\right)}{S(t, \vec{x})}
$$


and it is called the residence time probability distribution. Please observe that it is conditional to the clock time and the position $\vec{x}$. Integration over all the injection times, returns 1 , as it should be. The mean age of parcels inside the volume around $x$ is:

$$
\langle\operatorname{age}(t, \vec{x})\rangle=\int_{-\infty}^{t} \int_{\partial \Omega^{+}\left(t_{i n}\right)}\left(t-t_{i n}\right) p\left(t_{i n}, \vec{x}_{i n} \mid t, \vec{x}\right) d \vec{x}_{i n} d t_{i n}
$$

From the Lagrangian point of view, it is $\vec{x}_{i n}=\vec{x}_{i n}\left(t_{i n}\right)$. The determination of the domain $\partial \Omega^{+}=$ $\partial \Omega^{+}\left(t_{i n}\right)$, together with the knowledge of the Lagrangian trajectories $\vec{x}_{i n}=\vec{x}_{i n}\left(t_{i n}\right)$ determines the a switch to the Eulerian perspective over the parcels movements. Willing to eliminate the the dependence on space from the probability, to obtain the bulk probabilities, we have to perform and Integration over the domain $\Omega$, excluding the boundaries, and one over the entering boundary $\partial \Omega^{+}$to get all the input parcels. We obtain therefore what was called in (Rigon et al., 2016) residence time distribution:

$$
p\left(t_{i n} \mid t\right)=\int_{\Omega} \int_{\partial \Omega^{+}\left(t_{i n}\right)} p\left(t_{i n}, \vec{x}_{i n} \mid t, \vec{x}\right) p(\vec{x}) d x_{i n} d x
$$

However, it can be convenient to approach the mathematics from a different side, if we look at the previously cited paper (Rigon et al., 2016).

In that paper that refers to compartmental systems, the residence time is:

$$
p\left(t_{i n} \mid t\right):=\frac{s\left(t_{i n} \mid t\right)}{S(t)}=\frac{\int_{\partial \Omega^{+}} \int_{\Omega} s\left(t, \vec{x}, t_{i n}, \vec{x}_{i n}\right) d \vec{x}_{i n} d \vec{x}}{\int_{\partial \Omega^{+}} \int_{\Omega} \int_{-\infty}^{t} s\left(t, \vec{x}, t_{i n}, \vec{x}_{i n}\right) d \vec{x}_{i n} d \vec{x} d t_{i n}}
$$

and the equivalence has to be derived. To play with the conditional variables properly it is mandatory here, otherwise nothing works.

It is also interesting to explore what it is the fate of of outgoing fluxes. The volume (mass, number of moles) of parcels going out from the control volume at time $t$ is:

$$
Q(t):=\int_{\Omega^{-}} \int_{\partial \Omega^{+}} \int_{-\infty}^{t} s\left(t, \vec{x}, t_{i n}^{\prime}, \vec{x}_{i n}^{\prime}\right) d t_{i n}^{\prime} d \vec{x}_{i n} d \vec{x}
$$

\section{References}

Age-ranked hydrological budgets and a travel time description of catchment hydrology. (2016). Hydrology and Earth System Sciences, 20(12), 4929-4947. https://doi.org/10.5194/hess-20-4929-2016 